\title{
Correction: Chidamide and decitabine can synergistically induce apoptosis of Hodgkin lymphoma cells by up-regulating the expression of PU.1 and KLF4
}

\author{
Tao Jiang ${ }^{1,2, *}$, Fujue Wang ${ }^{1,4, *}$, Lianjie Hu ${ }^{1,4}$, Xiaomin Cheng ${ }^{1,3}$, Yuhuan Zheng ${ }^{1,4}$, \\ Ting Liu ${ }^{1,4}$ and Yongqian Jia ${ }^{1,4}$ \\ ${ }^{1}$ Department of Hematology, West China Hospital of Sichuan University, Chengdu 610041, Sichuan, China \\ ${ }^{2}$ Department of Hematology, Sichuan Academy of Medical Sciences \& Sichuan Provincial People's Hospital, Chengdu 610072, \\ Sichuan, China \\ ${ }^{3}$ Department of Hematology, Chengdu Military General Hospital, Chengdu 610083, Sichuan, China \\ ${ }^{4}$ Laboratory of Hematology, West China Hospital of Sichuan University, Tianfu Life Science Park, Chengdu 610041, Sichuan, \\ China \\ * These authors have contributed equally to this work
}

Published: April 20, 2018

Copyright: Jiang et al. This is an open-access article distributed under the terms of the Creative Commons Attribution License 3.0 (CC BY 3.0), which permits unrestricted use, distribution, and reproduction in any medium, provided the original author and source are credited.

This article has been corrected: The equal contribution note has been added to Drs. Jiang and Wang.

\section{Tao Jiang ${ }^{1,2, *}$, Fujue Wang ${ }^{1,4, *}$, Lianjie Hu ${ }^{1,4}$, Xiaomin Cheng ${ }^{1,3}$, Yuhuan Zheng ${ }^{1,4}$, Ting Liu ${ }^{1,4}$ and Yongqian Jia ${ }^{1,4}$ \\ * These authors have contributed equally to this work}

Original article: Oncotarget. 2017; 8:77586-77594. https://doi.org/10.18632/oncotarget.20659 